Canadian

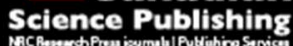

Applied Physiology, Nutrition, and Metabolism Physiologie appliquée, nutrition et métabolisme

\title{
Body composition changes affect energy cost of running during 12 months of specific diet and training in amateur athletes.
}

\begin{tabular}{|r|l|}
\hline Journal: & Applied Physiology, Nutrition, and Metabolism \\
\hline Manuscript ID: & apnm-2015-0023.R1 \\
\hline Manuscript Type: & Article \\
\hline Date Submitted by the Author: & 12 -Apr-2015 \\
\hline Lomplete List of Authors: & $\begin{array}{l}\text { Ghiani, Giovanna; University of Cagliari, Department of Medical Sciences } \\
\text { Marongiu, Elisabetta; University of Cagliari, Department of Medical } \\
\text { Sciences, Sports Physiology Lab. } \\
\text { Melis, Franco; University of Cagliari, Department of Medical Sciences } \\
\text { Angioni, Giuseppina; University of Cagliari, Department of Medical Sciences } \\
\text { Sanna, Irene; University of Cagliari, Department of Medical Sciences } \\
\text { Loi, Andrea; University of Cagliari, Department of Medical Sciences, Sports } \\
\text { Physiology Lab. } \\
\text { Pusceddu, Matteo; university of cagliari, department of medical science, } \\
\text { sport physiology Lab. } \\
\text { Pinna, Virginia; University of Cagliari, Department of Medical Sciences } \\
\text { Crisafulli, Antonio; University of Cagliari, Department of Medical Sciences } \\
\text { Tocco, Filippo; University of Cagliari, Department of Medical Sciences }\end{array}$ \\
\hline Keyword: & \begin{tabular}{l} 
diet , energy cost of motion, fat mass, free fat mass, running program \\
\hline
\end{tabular} \\
\hline
\end{tabular}

\section{SCHOLARONE}

Manuscripts 
1 Body composition changes affect energy cost of running during 12 months of specific

3

11 Correspondence to:

12 Filippo Tocco. ${ }^{1}$

\section{Giovanna Ghiani}

Giovanna Ghiani, ${ }^{1}$ Elisabetta Marongiu, ${ }^{1}$ Franco Melis, ${ }^{1}$ Giuseppina Angioni, ${ }^{1}$ Irene Sanna, ${ }^{1}$ Andrea Loi, ${ }^{1}$ Matteo Pusceddu, ${ }^{1}$ Virginia Pinna, ${ }^{1}$ Antonio Crisafulli, ${ }^{1}$ and

${ }^{1}$ Department of Medical Sciences, School of Sport Medicine, Sport Physiology Lab., University of Cagliari, Italy

Department of Medical Sciences,

School of Sport Medicine, Sport Physiology Lab., University of Cagliari, Italy

Via Porcell 4, 09124 Cagliari (Italy)

Tel +390706758927

Fax +390706758917

E-mail: giovanna.ghiani@me.com 


\section{Abstract}

Considering the relation between body weight-composition and energy cost of running, we tested the hypothesis that modifying body composition by means of a combined protocol of specific diet and training, the energy cost of motion $\left(\mathrm{C}_{\mathrm{r}}\right)$ may be reduced. 45 healthy and normal-weight subjects were divided into three groups that performed a different treatment: the first group attended only a dietary protocol (D), the second only a running program (R) and the third both $(\mathrm{R} \& \mathrm{D})$. Each subject underwent three anthropometric and exercise evaluation tests during one year (at T0, T6, T12) to assess body composition and $\mathrm{C}_{\mathrm{r}}$ adjustments. The mean fat mass (FM) values were reduced in $R \& D$ from $12.0 \pm 4.0$ to $10.4 \pm 3.0 \mathrm{~kg}(p<0.05 \mathrm{~T} 0$ vs. T12) and in the D group from $14.2 \pm 5.8$ to $11.6 \pm 4.7 \mathrm{~kg}(p<0.05$ T0 vs. T12). Conversely, the mean fat free mass values increased in R\&D (from $56.3 \pm 8.8$ to $58.3 \pm 9.8 \mathrm{~kg}, p<0.05 \mathrm{~T} 0$ vs. T12) and in the D group (from $50.6 \pm 13.2$ to $52.9 \pm 13.6 \mathrm{~kg}$, $p<0.05 \mathrm{~T} 0$ vs. T12). The mean $\mathrm{Cr}$ values of the 2 groups were significantly modified throughout the one year protocol $\left(1.48 \pm 0.16\right.$ and $1.40 \pm 0.15 \mathrm{kcal} \cdot \mathrm{kg}^{-\mathrm{b}} \cdot \mathrm{km}^{-1}$ in the $\mathrm{R} \& \mathrm{D}$ group at T0 and T12 respectively; $1.83 \pm 0.17$ and $1.76 \pm 0.23 \mathrm{kcal} \cdot \mathrm{kg}^{-\mathrm{b}} \cdot \mathrm{km}^{-1}$ in D group at T0 to $\mathrm{T} 12$ respectively). The $\mathrm{R} \& \mathrm{D}$ and $\mathrm{D}$ groups that underwent the diet protocol had a positive change in body composition during the year (FM-fat free mass ratio decline) which determined a Cr reduction.

Keywords: Diet; body composition; energy cost of motion; fat mass; free fat mass; running program. 


\section{Introduction}

It is well known that in endurance running, athletes with the lowest body mass (BM) generally achieve the best results (Sedeaud 2014). This is probably due to the fact that generating the force to support body weight is the primary determinant of the metabolic cost of running (Farley and McMahon 1992; Lacour and Bourdin 2015). Especially a low percentage of fat mass (FM) characterizes middle and long-distance runners and probably plays a pivotal role in their performances (Kong and de Heer 2008). Indeed, the gender differences of body fat content appears to be one of the more important factors related to the better results of male runners (Wilmore et al. 1977), and if male and female runners are matched on ability in the same distance, they show a similar percentage of FM (Pate et al. 1995). A recent study shows that in obese subjects $\mathrm{Cr}$ appears to be constant rather than rising with increasing $\mathrm{BM}$, probably because elastic tissue can adapt to this increment, thus maintaining its ability to store elastic energy (Taboga et al. 2012). On the other hand, Teunissen and coll. (2007) found that body mass reduction caused a decrease in net metabolic rate although not proportionally. Nevertheless, the authors of the aforementioned study reduced body mass by means of a harness system while not modifying body composition. Theoretically, inducing a shift between FM versus fat free mass, because of augmented body ability to store and release elastic energy, cost of running ( $\mathrm{Cr}$ ) should be reduced.

Metabolic measurement is still the criterion for analysis of endurance performance in running (Lacour and Bourdin 2015). Besides, the way in which different body size and anthropometrical characteristics impact performance has been recently investigated in runners, highlighting the need to taking into account the role of allometric scaling in the relationship between metabolic $\mathrm{Cr}$ and the running performance (Tartaruga et al. 2010; 
66 Tartaruga et al. 2013a). In the light of the information reported above and considering that

67 knowledge of relations between body mass-composition changes and the energy cost of

68 running is rather scanty, we tested the hypothesis that $\mathrm{Cr}$ could be reduced by modifying

69 body composition with a specific diet and running program. To do this, the running

70 efficiency of 45 subjects (divided in 3 groups that performed a different treatment) was

71 monitored through one year by means of metabolic measurements, also taking into account

72 the influence of allometric scaling (Tartaruga et al. 2013b). 
73 Materials and Methods

$74 \quad$ Subjects

7545 healthy and normal weight subjects (average BM index was 23.6 \pm 2.3 SD), 29 males and

7616 females, were enrolled. They were recruited with an advice on facebook. Inclusion

77 criteria were: a) to have an active lifestyle and practice running or other sport activities at

78

79

80

81

82

83

84

85

86

87

88

89

90

91

92

93

94

95

96

recreational level for at least three days a week, since at least five years; b) to have no specific dietary regimen; c) to have no metabolic diseases.

Anthropometric and metabolic characteristics of participants at the start of the study are shown in Table 1. All anthropometric measurements were taken using the Anthropometric Standardization Reference Manual by Lohman et al. (1988).

All participants were interviewed about their clinical history, so as to exclude any medication or drug treatment that could influence their metabolism. They were informed about the protocol and written consent was obtained. The study was approved by the local ethics committee and was carried out in accordance with the Helsinki Declaration.

\section{Experimental design}

Participants were divided in three groups: a group (no runners) attended only a dietary protocol (D), a group attended only a running program (R) and the last one (R\&D), both.

Each subject was measured anthropometrically and performed a treadmill test to assess body composition and Cr changes at entry (T0), after six months (T6) and finally after one year from entry (T12).

Anthropometric and diet approach.

For each subject basic anthropometric measurements including body mass (BM, kg), height, and body composition were taken. Body surface area (BSA) was also calculated by means of the Mosteller's formula (Mosteller 1987). In detail, body composition was 
97 assessed by means of the plicometry method (triceps, biceps, sub scapular, and supra iliac 118 (see Table 2).

119 skin folds). The skin fold thickness was measured with a Lange skin fold caliper (Beta technology) and FM \% was predicted by using the Durnin and Womersley (1974) skin fold thickness equation. From the difference between BM and FM expressed in $\mathrm{kg}$, fat free mass (kg) was calculated. As intertester variability is the major source of error in skin fold measurements all skin folds were taken on the right side of the body by the same experienced technician each time. Intratester reliability check was conducted on 16 subjects (8 females and 8 males) prior to testing and no significant differences were observed between the 2 trials for the sum of 4 skin folds. Moreover all measurements were always taken at the same time of the day and the participants were instructed to abstain from exercise prior to their test and avoid alcohol and caffeinated beverages $24 \mathrm{~h}$ prior to the test. In addition, participants were asked not to drink any fluid $4 \mathrm{~h}$ prior to their test and urinate within 30 min of test. It was checked that all female participants were in the same stage of their menstrual cycle during days of data collection. Skin fold thickness was taken three times and the average value was used in the equation.

Food frequency questionnaire.

Each subject was asked to fill in a semi-quantitative food-frequency questionnaire (Fidanza et al. 1995) to assess their usual intake, both in calories and nutrients. The questionnaire consists of 16 printed forms and 16 pages with colored photos of the most common foods and courses in the Italian diet. Instructions are included and the questionnaire was repeated at each control (at T6 and T12) to check the adherence and the compliance to the given diet

Energy Expenditure. 
120 A multisensory accelerometer (SenseWear Pro armband, Bodymedia, Inc., Pittsburgh, PA,

121 USA) which was worn on the upper right arm was used to assess subjects' daily energy

122 expenditure (EE). The device estimates EE using measurements from a biaxial

123 accelerometer and sensors that quantify galvanic skin response, heat flux and skin

124 temperature. The biaxial accelerometer records the number of steps and the duration of

125 physical activity (Drenowatz and Eisenmann 2011). The physical activity level (PAL) of

126 each participant was calculated by dividing the total daily energy expenditure by energy

127 expenditure during sleep (Sijtsma et al. 2013).

128 Dietary protocol.

129 A personalized diet providing all the essential energy and nutrients required was given to

$130 \mathrm{R} \& \mathrm{D}$ and $\mathrm{D}$ groups. The diet provided a personalized number of calories amounting to

131 about $20 \%$ proteins, $50 \%$ carbohydrates and $30 \%$ fats. Total daily intake was divided into

132 three main meals: breakfast (30\%), lunch (45\%) and dinner (25\%). The choice of giving

133 athletes a breakfast richer than their usual one was made because recent studies show a

134 close link of daily meal distribution, obesity, mass and fat loss (Jakubowicz et al. 2013;

135 Wang et al. 2013).

136 Training program.

137 Groups $\mathrm{R}$ and $\mathrm{R} \& \mathrm{D}$ attended a running program of at least $40 \mathrm{~km}$ per week in outdoor conditions (no treadmill) to attain an adequate energy expenditure (Slentz et al. 2005). In

139 detail, participants were asked to perform 4 running sessions (about $10 \mathrm{~km}$ per session at 140 the same speed on which the $\mathrm{Cr}$ was calculated, see below) per week spaced by 1 day of 141 rest. The goal was to reach a total energy expenditure of about 400-600 kcal per day in 142 order to evoke an adequate increase of metabolism and fat free mass (Zuti and Goldin 
143 1976). To check the adherence and the compliance to the training program participants

144 recorded the distance and the speed of training sessions by a standard GPS chronometer.

145 Treadmill tests.

146 Each subject underwent a preliminary incremental treadmill running test to volitional

147 exhaustion (RunRace, Tecnogym, Forlì, Italy) to assess his anaerobic threshold (AT). After

$1483 \mathrm{~min}$ of rest in the standing position on the treadmill, the subject started to run at $6 \mathrm{~km} \mathrm{~h}^{-1}$

149 and speed was increased by $1 \mathrm{~km} \cdot \mathrm{h}^{-1}$ every 3 minutes, until exhaustion. The treadmill

150 gradient was maintained at $1 \%$ throughout steps to reflect the energy cost of outdoor

151 running (Jones and Doust 1996). Achievement of maximal oxygen uptake $\left(\mathrm{VO}_{2} \mathrm{max}\right)$ was

152 considered as the attainment of at least two of the following criteria: 1) a plateau in $\mathrm{VO}_{2}$,

153 despite increasing speed; 2) a respiratory exchange ratio $>1.10$; and 3 ) a heart rate \pm 10

154 beats $\cdot \mathrm{min}^{-1}$ of predicted maximum heart rate (HR) calculated as 220-age (Howley et al.

155 1995). The ventilatory equivalent method was used to determine AT (Davis et al. 1979).

156 Briefly, AT was assessed as an increase in ventilatory equivalent for oxygen, [i.e.

157 pulmonary ventilation $\left(\mathrm{V}_{\mathrm{E}}\right)$ and oxygen consumption $\left(\mathrm{VO}_{2}\right)$ ratio, $\left.\left(\mathrm{V}_{\mathrm{E}} / \mathrm{VO}_{2}\right)\right]$ with no

158 associated increase in the ventilatory equivalent for carbon dioxide [i.e. pulmonary

159 ventilation $\left(\mathrm{V}_{\mathrm{E}}\right)$ and carbon dioxide output $\left(\mathrm{VCO}_{2}\right)$ ratio, $\left.\left(\mathrm{V}_{\mathrm{E}} / \mathrm{VCO}_{2}\right)\right]$.

160 After at least one week each participant performed a specific treadmill test to calculate the

161 energy cost of running. After a warm-up, participants ran at a speed $1 \mathrm{~km} \cdot \mathrm{h}^{-1}$ below the AT

162 for 5 minutes to obtain a steady-state condition to calculate $\mathrm{Cr}$. The fixed speed was

163 unchanged in T0, T6 and T12 (values ranged from 8 to $13 \mathrm{~km} \cdot \mathrm{h}^{-1}$ among subjects). In our

164 purpose, since within this range the step frequency remains about constant (Cavagna 2010),

165 an high running efficiency (because of our treatment) would occur probably indicating an 
166 augment of elastic recoil energy from the stretched contracted muscle-tendon units of 167 subjects. In this case the Cr should be reduced.

168 Assessment of respiratory variables. Throughout tests athletes wore a portable metabolic 169 system (MedGraphics VO2000, St. Paul, Minneapolis, USA) for pulmonary gas exchange.

170 This system is able to provide a 3-breath average of variables through telemetric

171 transmission and it has been demonstrated to be reliable and have a good agreement

172 compared to a standard metabolic cart for laboratory use (Crouter et al. 2006). Prior to

173 testing, the VO2000 was calibrated according to the manufacturer's instructions. By means

174 of the VO2000 the following parameters were assessed: oxygen uptake $\left(\mathrm{VO}_{2}\right)$, carbon

175 dioxide production $\left(\mathrm{VCO}_{2}\right)$, pulmonary ventilation $\left(\mathrm{V}_{\mathrm{E}}\right)$.

176 Measurement of aerobic energy expenditure. The aerobic $\mathrm{Cr}\left(\mathrm{kcal} \cdot \mathrm{kg}^{-1} \cdot \mathrm{km}^{-1}\right)$ was

177 calculated by dividing the average oxygen uptake (indexed by BM) by the distance covered

178 in that time (Fletcher et al. 2009). By utilizing the Weir equation (Weir 1949; Mansell et al.

179 1990), this quantity was multiplied using the following equation: $\mathrm{Cr}=$

$1803.941 \cdot \mathrm{VO}_{2}+1.106 \cdot \mathrm{VCO}_{2}$ to obtain $\mathrm{kcal}$ from oxygen uptake. This equation was used when

181 the respiratory exchange ratio (RER) was $<1$ i.e. it was assumed that all aerobic energy

182 derived from carbohydrate and fat oxidation. As reported above, $\mathrm{Cr}$ was measured during 183 the last minute of five performed at a speed of $1 \mathrm{~km} \cdot \mathrm{h}^{-1}$ slower than AT speed. Moreover, 184 since previous researches have underlined that an arbitrary use of the $\mathrm{ml} \cdot \mathrm{kg}^{-1} \cdot \mathrm{min}^{-1}$ is 185 inappropriate for the purpose of comparing $\mathrm{Cr}$ between subjects with different body 186 characteristics (Markovic et al. 2007; Tartaruga et al. 2013b), the effect of allometric 187 scaling on $\mathrm{Cr}$ was also investigated. The relationship of one variable $\left(\mathrm{VO}_{2}\right)$ with respect to 188 another $(\mathrm{BM})$ can be summarized by the allometric coefficient of the equation of the 189 relationship. To obtain this allometric coefficient the first variable could be expressed as a 
190 function of the second using the general exponential equation (1): $y=a x^{b}$ where $y$ is the

191 absolute $\mathrm{VO}_{2}, x$ is the BM, $a$ is a constant characteristic for the organism and $b$ is the

192 allometric or scaling exponent. This exponential function can be changed into a linear

193 function (equation 2): $\log y=\log a+b \log x$, where $b$, the slope of the line, is the

194 allometric coefficient. Thus, the log-log plot between subjects' $\mathrm{VO}_{2}$ and BM at T0-T6 and

195 T12 was used to determine the allometric exponent for scaling the subjects' Cr (Fig. 5).

196 Statistical analysis

197 The normality assumption was checked using the Kolmogorov-Smirnov test. The $\alpha$ level

198 was set at $\mathrm{p}<0.05$. Comparisons between tests were performed using the repeated

199 measures analysis of variance (ANOVA) followed by Neuman- Keuls post hoc when

200 appropriate. Differences between groups were analyzed by means of the two-way ANOVA

201 for repeated measures followed by Tukey post-hoc when appropriate. Inter-group time

202 related differences (mean \pm SD) were shown as delta $(\Delta)$ values between T0 and T6 and

203 between T0 and T12 and assessed by means of one-way ANOVA followed by Tukey post-

204 hoc when appropriate. Statistics were produced utilizing commercially available software

205 (Graph-Pad, Prism). The $\alpha$ level was set at $\mathrm{p}<0.05$ in all cases. 


\section{Results}

208 Absolute Cr, BM, FM and fat free mass values of each group, measured at the beginning of 209 the study (T0), after six months (T6) and one year later (T12) are shown in Table 3.

210 All 3 groups displayed significant reduction in $\mathrm{Cr}$ over the entire year without no

211 significant loss or gain in BM over the entire year. R\&D body composition significantly

212 changed after 6 and 12 months. In fact, while their FM was reduced by $15 \%$ from T0 to T6

213 and T12 $(p<0.05)$, the fat free mass increased by $3 \%$ at T6 and T12 $(p<0.05)$.

214 The R group body composition did not substantially change during the year. However, their

215 FM slightly decreased at T6 but increased at T12 ( $p<0.05$ vs. T6), changing back to T0

216 values. In contrast, fat free mass slightly increased at T6 and declined at T12 ( $p<0.05$ vs.

217 T6), going back to T0 values.

218 The D group body composition was significantly modified both in FM and fat free mass

219 because the former decreased by $15 \%$ at T6 and $9 \%$ at T12 ( $p<0.05$ vs. T0) and the latter

220 increased by $3.4 \%$ at T6 and $4 \%$ at T12 ( $p<0.05$ vs. T0).

221 Figures from 1 to 4 show $\Delta$ values for each parameter of T0, T6 and T12 to evaluate

222 differences among the three groups following the three different regimens.

223 In detail, no significant difference in $\Delta \mathrm{Cr}$ was found after 6 months (Fig. 1, top panel)

224 while after 12 months $\Delta \mathrm{Cr}$ was reduced in the $\mathrm{R} \& \mathrm{D}$ and $\mathrm{D}$ groups (Figure 1, bottom

225 panel). Figure 2 did not display any difference in $\Delta$ FM after 6 months (top panel) while at

226 T12 the R group FM increased by $0.48 \pm 1.18 \mathrm{Kg}$ ( $p<0.05$ vs. R\&D and D groups, which

227 showed a decreasing trend). On the contrary, the $\Delta$ values of fat free mass increased in the

228 R\&D and D groups both after 6 and 12 months, while after one year the R group showed a 229 fat free mass decrement $(-0.37 \pm 0.65 \mathrm{~kg}, p<0.05$ vs. $\mathrm{R} \& \mathrm{D}$ and $\mathrm{D}$ groups $)$ as shown in Fig. 3 . 
230 Consequent to FM and fat free mass opposite changes, the $\Delta \mathrm{BM}$ values were unmodified

231 both after 6 and 12 months in all three groups (Fig. 4, top and bottom panels).

232 
233 Discussion

234 The aim of the study was to verify whether a one-year combined protocol of running and an 235 isocaloric high-intake breakfast diet could modify body composition and this, in turn, 236 evoking a Cr reduction. Our hypothesis was based on a presumable higher muscular tissue 237 capacity to store elastic energy than adipose tissue. Athletes with an augmented fat free mass can potentially store more elastic strain energy because their stronger muscles can impose higher tendon stresses. To the best of our knowledge, this is the first study that analyzed the relationship between the fat free mass-FM ratio and the energy cost of running on a treadmill.

As expected, the dietary intervention modified the body composition in the R\&D and D groups: the isocaloric high-intake breakfast diet decreased the FM and increased the fat free mass although without significant changes in BM. A recent analysis by Garaulet and Gómez-Abellán (2014) highlighted that much evidence exists about the importance of the timing of food and macronutrient distribution. Besides, subjects assigned to a high caloric intake during breakfast lost significantly more mass than ones assigned to high caloric intake during dinner (Jakubowics et al. 2013). The latter authors and others (Wang et al. 2013) described the presence of an active circadian clock in different organs related to food intake, which influences the changes in BM and body composition. During our one-year study we detected FM and fat free mass adjustments only, without any change in BM. In any case, the two dieting groups showed a fat free mass gain and a FM loss while the R group displayed opposite changes. This outcome means that the specific diet successfully modified body composition while exercise only did not. It is important to note that our results are quite different from those of the aforementioned studies by Jakubowicz et coll. (2013) where the isocaloric diet caused a mass loss. On the other hand, it is to be 
257 considered that our sample was represented by normal-mass amateur athletes and not by

258 obese subjects.

259 In agreement to the initial hypothesis, the $\mathrm{Cr}$ after 12 months had diminished with respect

260 to T0 and difference among the three groups was found. In fact the two groups that

261 followed a dietary regimen showed a larger $\mathrm{Cr}$ decrease (Fig. 1, bottom panel). As

262 expected, the shift between FM and fat free mass was able to increase the total ability to

263 store and release elastic energy during running and decrease $\mathrm{Cr}$ at a given speed.

264 Noteworthy, when Cr was calculated without groups' specific allometric scaling (data not

265 shown) this reduction was not found and in that case the results suggested that $\mathrm{Cr}$ is not

266 dependent on body composition changes evoked by our protocol. Hence the need to taking

267 into account the role of allometric scaling in the relationship between metabolic $\mathrm{Cr}$ and the

268 performance in this kind of studies is strongly recommended (Tartaruga et al. 2010;

269 Tartaruga et al. 2013a).

270 Previous past and recent studies (Margaria et al. 1963; Ferretti et al. 2011), have shown that

271 the energy cost of running does not appear to be easily modified. The reference model by

272 Margaria et al. (1963) pointed out that the mechanical efficiency of running is quite stable

273 in athletes, that is, training does not lead to great improvement. Therefore, Cr values are

274 only 5-7\% lower in athletes than in untrained subjects. The analysis by Ferretti et al. (2011)

275 reiterated that $\mathrm{Cr}$ is "barely" modifiable in running as concerns intrinsic human

276 characteristics, while the evolution of equipment (track and shoe elasticity) has

277 progressively contributed to improving performance. However, the above-mentioned points

278 of views arise with the classical assessment of Cr, i.e. without considering the impact of

279 allometric scaling. In our study, taking into account the different anthropometric factors

280 between groups a different outcome was found. 
281 In our setting, no significant changes in BM was found in any of the 3 groups without that, 282 however, this would change the expected results. Considering the role of BM changes, 283 Farley and McMahon (1992) suggested that the metabolic cost of running is proportional to 284 body mass, while other authors recently found that the reduction of body mass with a 285 harness system decreased the Cr even though not in a proportional way (Teunissen et al. 286 2007). In any case, it appears that Cr primarily depends on BM. On the contrary a recent 287 review by Lacour and Bourdin (2015) pointed out on the role of others factors, like leg 288 architecture and elastic energy storage and reuse, as pivotal in the running performance. 289 Conclusions. On the basis of our findings, the induced shift between FM and fat free mass 290 with an augmented of the latter (and with BM unchanged) in R\&D and D groups led to a $\mathrm{Cr}$ 291 reduction during running. Thus the $\mathrm{Cr}$ appears to be strongly related to body composition at 292 least within the range of changes evoked by our protocol. 
293 Acknowledgements

294 We are grateful to Mr. Nardino Degortes for his technical assistance to the athletes

295 undergoing the running tests.

296 We are also grateful to the Cagliari Marathon Club for its help in carrying

297 out the study.

298

299 Disclosures

300 The Authors have no conflicts of interest to disclose with regards this manuscript.

301

302 Author contributions

303 G.G., F.M., A.C. and F.T. participated in the design of the experimental protocol and in the

304 discussion of results. G.G., E.M., G.A., I.S., A.L., M.P., and V.P. performed most of the

305 measurements and data analyses. G.G. and A.C. also drafted and revised the manuscript.

306 G.G. and G.A. recruited volunteers. E.M. and F.M. carried out medical supervision. F.T.

307 was responsible for all stages of the study and revised the manuscript.

308 
309

310

311

312

313

314

\section{References}

Cavagna, G.A. 2010. Symmetry and asymmetry in bouncing gaits. Symmetry 2: 12701321.

Crouter, S.E., Antczak, A., Hudak, J.R., DellaValle, D.M., and Haas, J.D. 2006. Accuracy and reliability of the ParvoMedics TrueOne 2400 and MedGraphics VO2000 metabolic systems. Eur J Appl Physiol 98: 139-151.

Davis, J.A., Frank, M.H., Whipp, B.J., and Wasserman, K. 1979. Anaerobic threshold alterations caused by endurance training in middle age man. J Appl Physiol 46:1039-1046.

Drenowatz, C., and Eisenmann, J.C. 2011.Validation of the SenseWear Armband at high intensity exercise. Eur J Appl Physiol 111: 883-887.

Durnin, J., and Womersley, J. 1974. Body fat assessed from total body density and its estimation from skinfold thickness: measurements on 481 men and women aged from 16 to 72 years. Br J Nutr 32: 77-97.

Farley, C.T., and McMahon, T.A. 1992. Energetics of walking and running - insights from simulated reduced-gravity experiments. J Appl Physiol 73: 2709 -2712.

Ferretti, G., Bringard, A., Perini, R. 2011. An analysis of performance in human locomotion. Eur J Appl Physiol 111: 391-401.

Fidanza, F., Gentile, M.,G., and Porrini, M.A. 1995. Self-administered semi quantitative food-frequency questionnaire with optical reading and its concurrent validation. Eur J Epidemiol 11: 163-170.

Fletcher, J.R., Esau, S.P., and Macintosh, B.R. 2009. Economy of running: beyond the measurement of oxygen uptake. J Appl Physiol 107: 1068-1075. 
331 Garaulet, M., and Gomez-Abellan, P. 2014. Timing of food intake and obesity: A novel

332 association. Physiology \& Behavior 134: 44-50.

333 Howley, E.T., Bassett, D.R., and Welch, H.G. 1995. Criteria for maximal oxygen uptake:

334 review and commentary. Med Sci Sports Exerc 27: 1292-1301.

335 Jakubowicz, D., Barnea, M., Wainstein, J., and Froy, O. 2013. High caloric intake at

336 breakfast vs. dinner differentially influences mass loss of overmass and obese women.

337 Obesity 21: 2504-2512.

338 Jones, A., and Doust, J. 1996. A 1\% treadmill grade most accurately reflects the energetic

339 cost of outdoor running. J Sports Sci 14: 321-327.

340 Kong, P.W., and de Heer, H. 2008. Anthropometric, gait and strength characteristics of

341 Kenyan distance runners. J Sports Sci Med 7(4): 499-504.

342 Lacour, J.R., and Bourdin M. 2015. Factors affecting the energy cost of level running at 343 submaximal speed. Eur J Appl Physiol 115(4): 651-673.

344 Lohman, T.G., Roche, A., and Martorell, R. 1988. Anthropometric standardization

345 Reference Manual. Human Kinetics, Champaign, IL.

346 Margaria, R., Cerretelli, P., Aghemo, P., and Sassi, G. 1963. Energy cost of running. J Appl

347 Physiol 18: 367-370.

348 Mansell, P.I., Fellows, I.W., MacDonald, I.A., and Allison, S.P. 1990. Defect in

349 Thermoregulation in Malnutrition Reversed by Mass Gain. Physiological Mechanisms and

350 Clinical Importance. Q J Med 76(280): 817-829.

351 Markovic, G., Vucetic, V., and Nevill, A.M. 2007. Scaling behaviour of $\mathrm{VO}_{2}$ in athletes

352 and untrained individuals. Ann Hum Biol 34: 315-328. 
353 Mosteller, R.D. 1987. Simplified calculation of body-surface area. N Engl J Med 22:

$354 \quad 317(17): 1098$.

355 Pate, R.R., Barnes, C., and Miller, W. 1995. A physiological comparison of performance356 matched female and male distance runners. Res Q Exerc Sport 56: 245-250.

357 Sedeaud, A., Marc, A., Marck, A., Dor, F., Schipman, J., Dorsey, M., Haida, A., Berthelot, 358 G., and Toussaint, J.F. 2014. BMI, a performance parameter for speed improvement. PLoS 359 One. 25; 9(2): e90183. doi: 10.1371.

360 Sijtsma, A., Schierbeek, H., Goris, A.H.C., Joosten, K.F.M., van Kessel, I., Corpeleijn, E., 361 and Sauer, P. J.J. 2013. Validation of the tracmor D triaxial accelerometer to assess 362 physical activity in preschool children. Obesity 21: 1877-1883.

363 Slentz, C.A., Aiken, L.B., Houmard, J.A., Bales, C.W., Johnson, J.L., Tanner, C.J., Duscha, 364 B.D., and Kraus, W.E. 2005. Inactivity, exercise, and visceral fat. STRRIDE: a 365 randomized, controlled study of exercise intensity and amount. J Appl Physiol 99: 16133661618.

367 Taboga, P., Lazzer, S., Fessehatsion, R., Agosti, F., Sartorio, A., and di Prampero P.E. 368 2012. Energetics and mechanics of running men: the influence of body mass. Eur J Appl 369 Physiol 112: 4027-4033.

370 Tartaruga, M.P., de Medeiros, M.H., Alberton, C.L., Cadore, E.L., Peyré-Tartaruga, L.A., 371 Baptista, R.R., Coertjens, M., and Kruel, L.F.M. 2010. Application of the allometric scale 372 for the submaximal oxygen uptake in runners and rowers. Biol Sport 27: 297-300.

373 Tartaruga, M.P., Bolli Mota, C., Peyré-Tartaruga, L.A., Kruel, L.F.M., Vallier, J.M., and 374 Brisswalter, J. 2013a. Running efficiency and long-distance performance prediction: 375 Influence of allometric scaling. Science \& Sports 28: 165-171. 
376 Tartaruga, M.P., Brisswalter, J., Bolli Mota, C., Alberton, C.L., Gomeňuka, N.A., Peyré-

377 Tartaruga, L.A. 2013b. Mechanical work and long-distance performance prediction: the

378 influence of allometric scaling. J Hum Kinet 38: 73-82.

379 Teunissen, L.P.J, Grabowski, A., and Kram, R. 2007. Effects of independently altering

380 body weight and body mass on the metabolic cost of running. J Exp Biol 210: 4418-4427.

381 Troosters, T., Sciurba, F., Battaglia, S., Langer, D., Valluri, S.R., Martino, L., Benzo, R.,

382 Andre, D., Weisman, I., and Decramer, M. 2010. Physical inactivity in patients with

383 COPD, a controlled multi center pilot study. Respir Med 104(7):1005-1011.

384 Wang, J.B., Patterson, R.E., Ang, A., Emond, J.A., Shetty, N., and Arab, L. 2013. Timing

385 of energy intake during the day is associated with the risk of obesity in adults. J Hum Nutr

386 Diet 27: 255-262.

387 Weir, J.B. 1949. New methods for calculating metabolic rate with special reference to 388 protein metabolism. J Physiol 109:1-9.

389 Wilmore, J.H., Brown, C.H., and Davis. J.A. 1977. Body physique and composition of 390 female distance runner. Ann N Y Acad Sci 301: 764-776.

391 Zuti, W.B., and Golding, L.A. 1976. Comparing diet and exercise as weight reduction 392 tools. The Physician and Sports Medicine 4: 49-53. 
394 Table 1. Anthropometric and metabolic parameters of the three groups at the beginning of 395 the study

\begin{tabular}{|c|c|c|c|c|}
\hline & $\begin{array}{l}\text { RUNNING } \\
\text { AND DIET }\end{array}$ & $\begin{array}{c}\text { RUNNING } \\
\text { GROUP }\end{array}$ & DIET GROUP & $\begin{array}{l}\text { WHOLE } \\
\text { SAMPLE }\end{array}$ \\
\hline $\begin{array}{c}\text { AGE } \\
\text { (years) }\end{array}$ & $43.7 \pm 4.3$ & $43.9 \pm 3.0$ & $41.5 \pm 9.7$ & $41.1 \pm 7.5$ \\
\hline $\begin{array}{l}\text { BM } \\
(\mathrm{kg})\end{array}$ & $68.3 \pm 7.8$ & $64.8 \pm 9.4$ & $64.8 \pm 15.5$ & $66.5 \pm 10.3$ \\
\hline $\begin{array}{c}\text { HEIGHT } \\
(\mathrm{cm})\end{array}$ & $168.8 \pm 8.4$ & $167.3 \pm 7.7$ & $164.2 \pm 8.0$ & $167.3 \pm 8.2$ \\
\hline $\begin{array}{c}\text { BSA } \\
\left(\mathrm{m}^{2}\right)\end{array}$ & $1.6 \pm 0.01$ & $1.51 \pm 0.01$ & $1.48 \pm 0.01$ & $1.54 \pm 0.01$ \\
\hline $\begin{array}{l}\text { FM } \\
(\mathbf{k g})\end{array}$ & $12.0 \pm 4.0$ & $12.3 \pm 3.2$ & $14.2 \pm 5.8$ & $12.5 \pm 4.2$ \\
\hline $\begin{array}{c}\text { FAT FREE } \\
\text { MASS } \\
(\mathrm{kg})\end{array}$ & $56.3 \pm 8.75$ & $52.5 \pm 10.3$ & $50.6 \pm 13.2$ & $53.9 \pm 10.4$ \\
\hline $\begin{array}{c}\mathbf{P} \\
(\%)\end{array}$ & $15.4 \pm 2.7$ & $15.8 \pm 2.5$ & $15.5 \pm 2.0$ & $15.5 \pm 2.5$ \\
\hline $\begin{array}{c}\mathrm{L} \\
(\%)\end{array}$ & $37.2 \pm 5.7$ & $37.1 \pm 6.3$ & $35.5 \pm 4.2$ & $36.8 \pm 5.5$ \\
\hline $\begin{array}{c}\mathrm{C} \\
(\%)\end{array}$ & $47.4 \pm 7.1$ & $47.2 \pm 7.1$ & $49.0 \pm 4.8$ & $47.7 \pm 6.6$ \\
\hline $\begin{array}{c}\text { TEE } \\
\text { (kcal/24h) }\end{array}$ & $2733 \pm 443$ & $2656 \pm 386$ & $2747 \pm 503$ & $2653 \pm 443$ \\
\hline
\end{tabular}




\begin{tabular}{|l|l|l|l|l|}
\hline PAL & $1.7 \pm 0.2$ & $1.7 \pm 0.2$ & $1.6 \pm 0.1$ & $1.7 \pm 0.2$ \\
\hline
\end{tabular}

396

397 Values are Mean \pm SD. BM: body mass; BSA: body surface area; FM: fat mass; FFM: fat

398 free mass; FFQ: food frequency questionnaire; P: daily protein amount; L: daily lipid

399 amount; C: daily carbohydrate amount; TEE: total daily energy expenditure; PAL:

400 physical activity level.

401

402 Table 2. Compliance to the given diet: FFQ results at the beginning of the study and at T6 403 and T12.

\begin{tabular}{|c|c|c|c|}
\hline & RUNNING & RUNNING & DIET GROUP \\
\hline FFQ (T0) & AND DIET & GROUP & \\
\hline (kcal/24h) & $2299 \pm 753$ & $2393 \pm 1019$ & $2530 \pm 771$ \\
\hline FFQ (T6) & $2352 \pm 635$ & $2424 \pm 852$ & $2512 \pm 546$ \\
(kcal/24h) & & & $2523 \pm 611$ \\
\hline FFQ (T12) & $2235 \pm 563$ & $2476 \pm 987$ & \\
\hline (kcal/24h) & & & \\
\hline
\end{tabular}

404

405 Values are mean \pm SD; FFQ: food frequency questionnaire.

406 
407 Table 3. Groups' data measured at the beginning of the study (T0), after six months (T6) 408 and one year later (T12) 409

\begin{tabular}{|c|c|c|c|c|}
\hline & TIME & $\begin{array}{c}\text { RUNNING \& } \\
\text { DIET }\end{array}$ & RUNNING & DIET \\
\hline \multirow{3}{*}{$\begin{array}{c}\mathrm{Cr} \\
\left(\mathrm{kcal} \cdot \mathrm{kg}^{-\mathrm{b}} \cdot \mathrm{km}^{-1}\right)\end{array}$} & T0 & $1.48 \pm 0.16$ & $1.16 \pm 0.10$ & $1.83 \pm 0.17$ \\
\hline & T6 & $1.34 \pm 0.16^{*}$ & $1.05 \pm 0.11 *$ & $1.72 \pm 0,22 *$ \\
\hline & $\mathrm{T} 12$ & $1.40 \pm 0.15^{* \dagger}$ & $1.14 \pm 0.12 * \dagger$ & $1.76 \pm 0.23^{*} \dagger$ \\
\hline \multirow{3}{*}{$\begin{array}{l}\text { BM } \\
(\mathrm{kg})\end{array}$} & T0 & $68.3 \pm 7.8$ & $64.8 \pm 9.4$ & $64.8 \pm 15.5$ \\
\hline & T6 & $68.0 \pm 8.7$ & $64.6 \pm 9.6$ & $64.1 \pm 15.1$ \\
\hline & T12 & $68.5 \pm 9.0$ & $64.9 \pm 9.5$ & $65.5 \pm 15.7$ \\
\hline \multirow{3}{*}{$\begin{array}{l}\text { FM } \\
(\mathbf{k g})\end{array}$} & T0 & $12.0 \pm 4.0$ & $12.3 \pm 3.2$ & $14.2 \pm 5.8$ \\
\hline & T6 & $10.1 \pm 2.8^{*}$ & $11.4 \pm 2.4$ & $11.9 \pm 5.0 *$ \\
\hline & T12 & $10.4 \pm 3.0 *$ & $12.7 \pm 3.0 \dagger$ & $11.6 \pm 4.7^{*}$ \\
\hline \multirow[t]{3}{*}{ FAT FREE } & T0 & $56.3 \pm 8.75$ & $52.5 \pm 10.3$ & $50.6 \pm 13.2$ \\
\hline & T6 & $58.2 \pm 9.2 *$ & $53.0 \pm 10.0$ & $52.2 \pm 13.4^{*}$ \\
\hline & $\mathrm{T} 12$ & $58.3 \pm 9.8^{*}$ & $52.1 \pm 10.2 \dagger$ & $52.9 \pm 13.6^{*}$ \\
\hline
\end{tabular}

410

411 Cr: cost of running; BM: body mass; FM: fat mass; FAT FREE MASS: free fat mass.

412 Values are mean \pm SD. $*=p<0.05$ vs. T0; $\dagger=p<0.05$ vs. T6 413 
414 Figure Captions

415

416 Figure 1. Cost of running $(\mathrm{Cr})$ variation $(\Delta)$ after 6 months $\left(\mathrm{T}_{0}-\mathrm{T}_{6}\right.$, top panel) and 12

417 months $\left(\mathrm{T}_{0}-\mathrm{T}_{12}\right.$, bottom panel) in the three groups. $\mathrm{R} \& \mathrm{D}$ : running and diet group; $\mathrm{R}$ :

418 running group; D: diet group. ${ }^{*}=p<0,05$ vs. running group.

419

420

421

422

Figure 2. Fat Mass $(\mathrm{FM})$ variation $(\Delta)$ after 6 months $\left(\mathrm{T}_{0^{-}} \mathrm{T}_{6}\right.$, top panel) and 12 months $\left(\mathrm{T}_{0^{-}}\right.$ $\mathrm{T}_{12}$, bottom panel) in the three groups. $\mathrm{R} \& \mathrm{D}$ : running and diet group; R: running group; $\mathrm{D}$ : diet group. ${ }^{*}=p<0,05$ vs. running group

423

424

425

426 diet group. ${ }^{*}=p<0,05$ vs. running Group

427

Figure 5. Relationship between oxygen uptake expressed in absolute terms $\left(\mathrm{VO}_{2}\right)$ and body

Figure 4. Body Mass $(\mathrm{BM})$ variation $(\Delta)$ after 6 months $\left(\mathrm{T}_{0}-\mathrm{T}_{6}\right.$, top panel) and 12 months ( $\mathrm{T}_{0}-\mathrm{T}_{12}$, bottom panel) in the three groups. $\mathrm{R} \& \mathrm{D}$ : running and diet group; $\mathrm{R}$ : running group; D: diet group

434 mass by using allometric log-linear for 3 groups (R\&D, top panel; R, middle panel; D, bottom panel). Linear regression is shown with a $95 \%$ confidence interval. 
Cr T0-T6

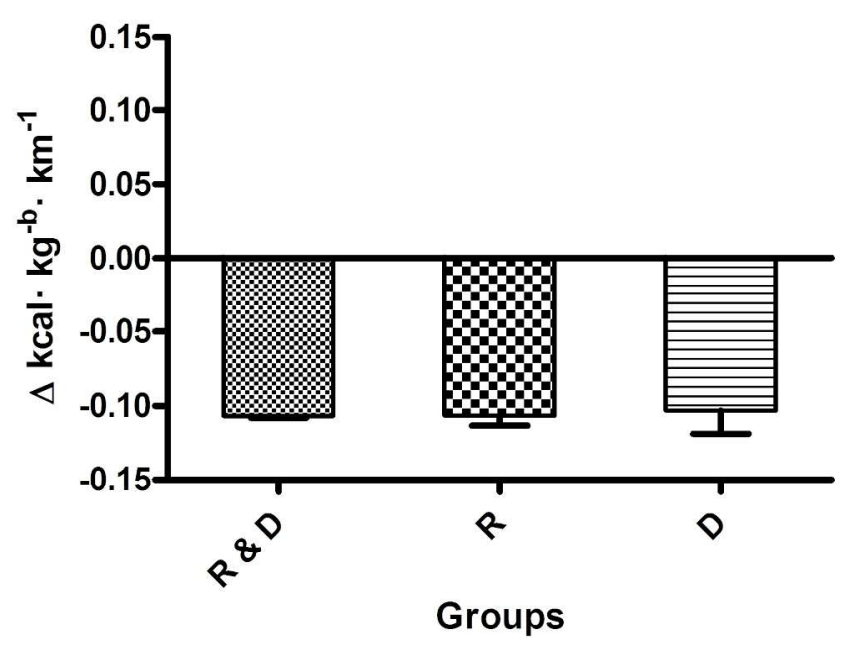

Cr T0-T12

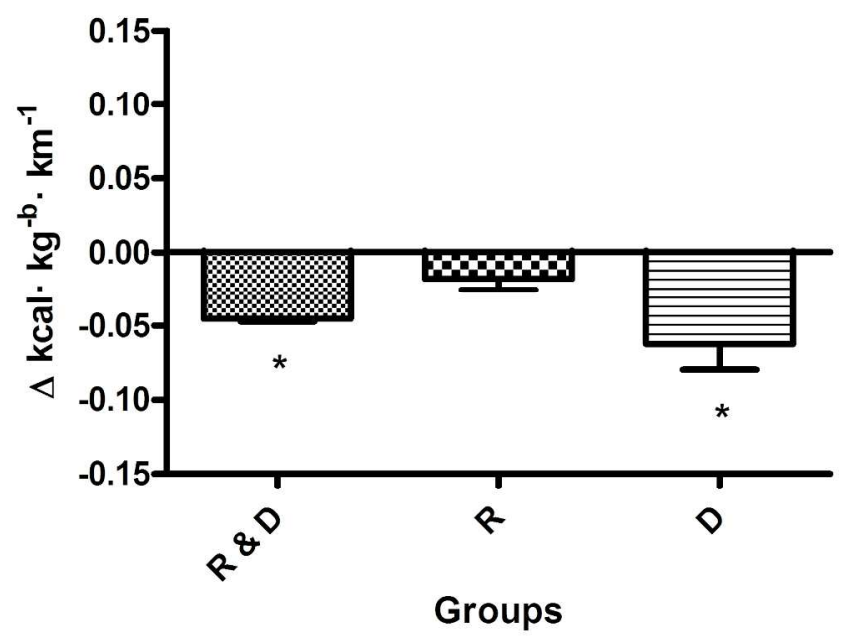

Figure 1. Cost of running $(\mathrm{Cr})$ variation $(\Delta)$ after 6 months (T0-T6, top panel) and 12 months (T0-T12, bottom panel) in the three groups. R\&D: running and diet group; R: running group; D: diet group. $*=p<0,05$ vs. running group. $156 \times 267 \mathrm{~mm}(300 \times 300 \mathrm{DPI})$ 
FM T0-T6

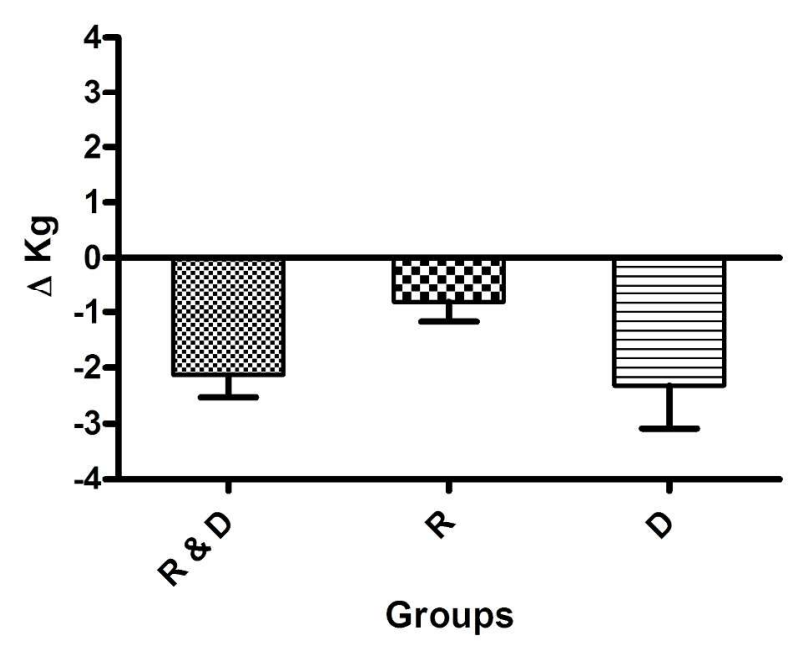

FM T0-T12

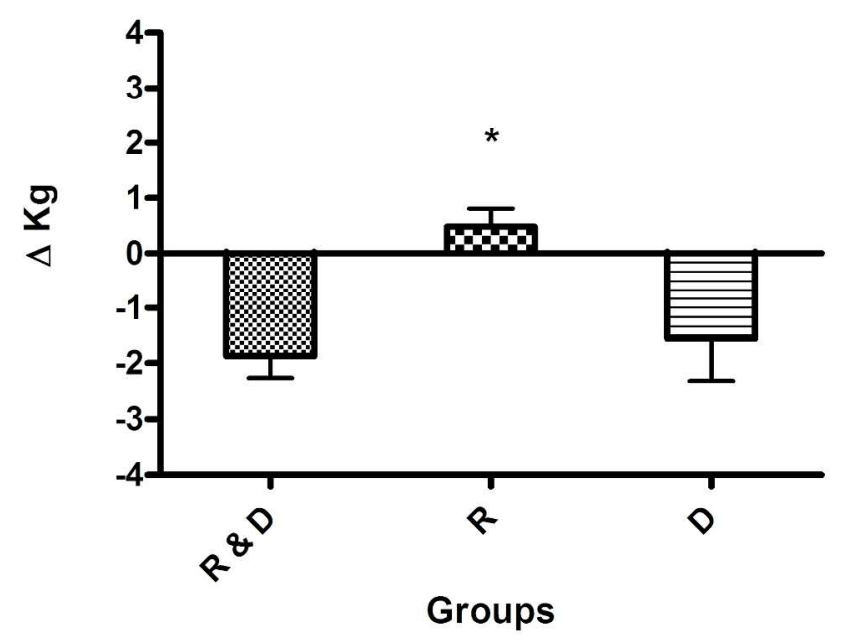

Figure 2. Fat Mass (FM) variation $(\Delta)$ after 6 months (T0-T6, top panel) and 12 months (T0-T12, bottom panel) in the three groups. R\&D: running and diet group; R: running group; $D$ : diet group. ${ }^{*}=p<0,05$ vs. running group $154 \times 267 \mathrm{~mm}(300 \times 300 \mathrm{DPI})$ 
FFM T0-T6

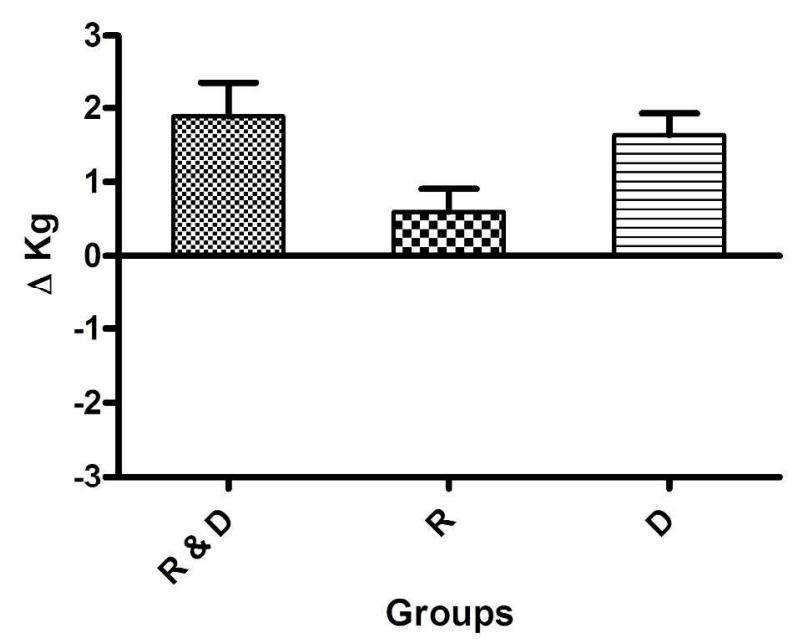

FFM T0-T12

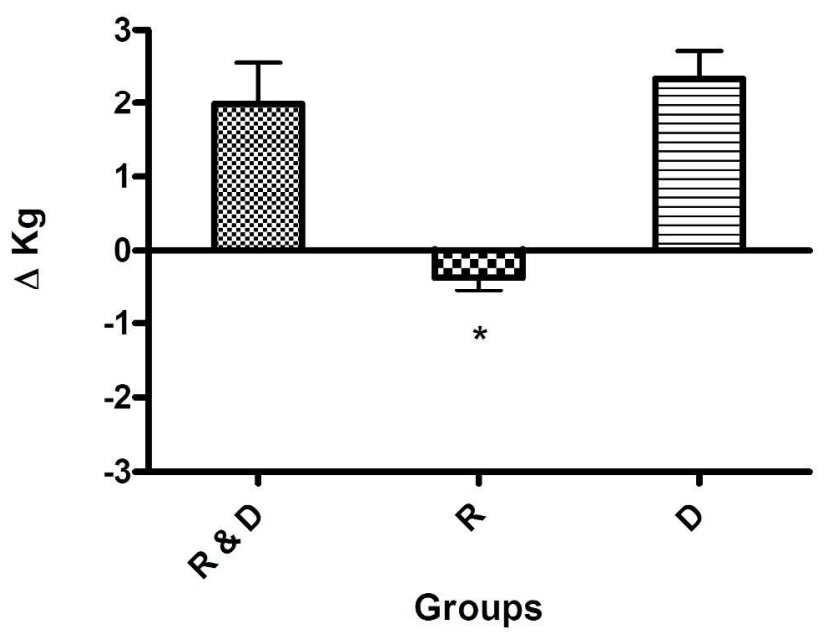

Figure 3. Fat Free Mass variation $(\Delta)$ after 6 months (T0-T6, top panel) and 12 months (T0-T12, bottom panel) in the three groups. R\&D: running and diet group; R: running group; $D$ : diet group. ${ }^{*}=p<0,05$ vs. running Group

$154 \times 267 \mathrm{~mm}(300 \times 300 \mathrm{DPI})$ 


\section{BM T0-T6}

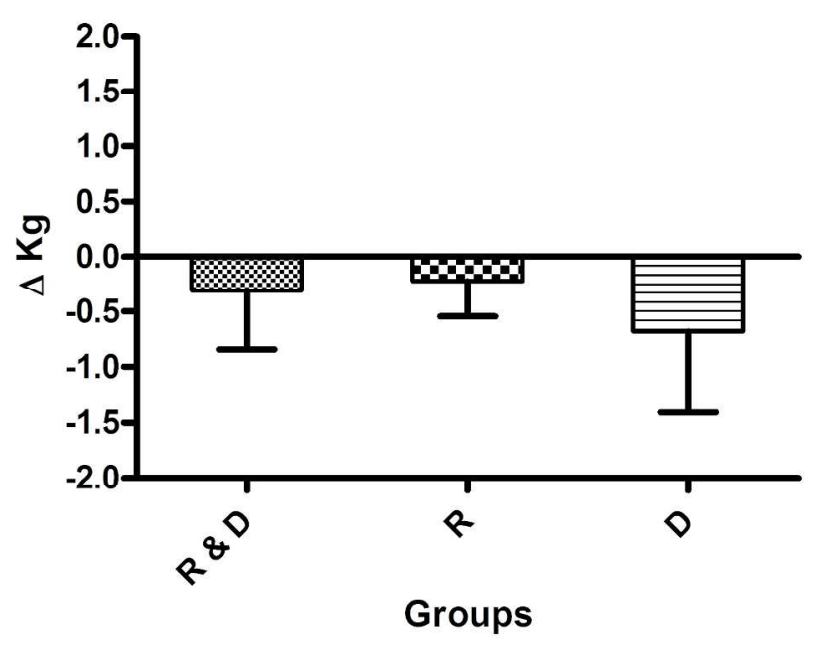

BM T0-T12

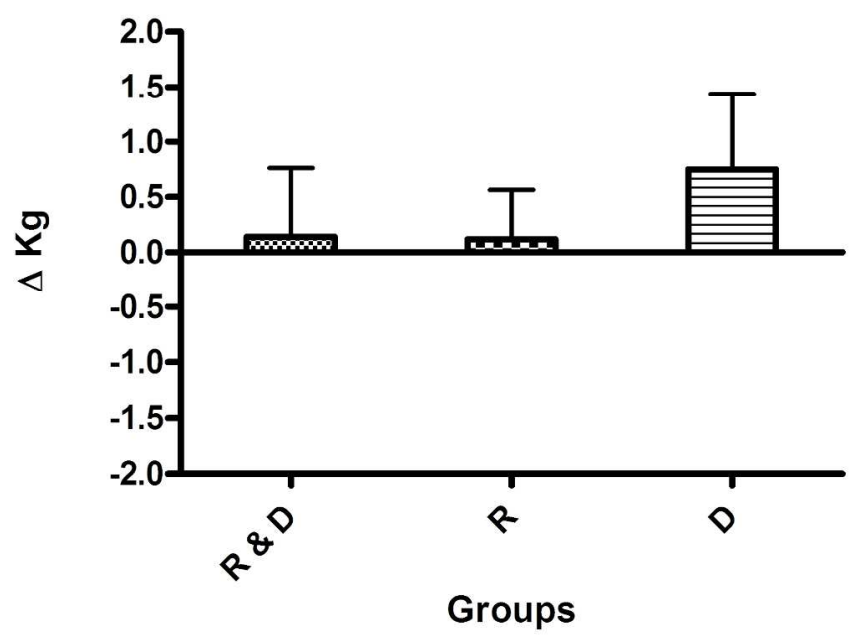

Figure 4. Body Mass (BM) variation $(\Delta)$ after 6 months (T0-T6, top panel) and 12 months (T0-T12, bottom panel) in the three groups. R\&D: running and diet group; R: running group; D: diet group $159 \times 267 \mathrm{~mm}(300 \times 300 \mathrm{DPI})$ 
R\&D

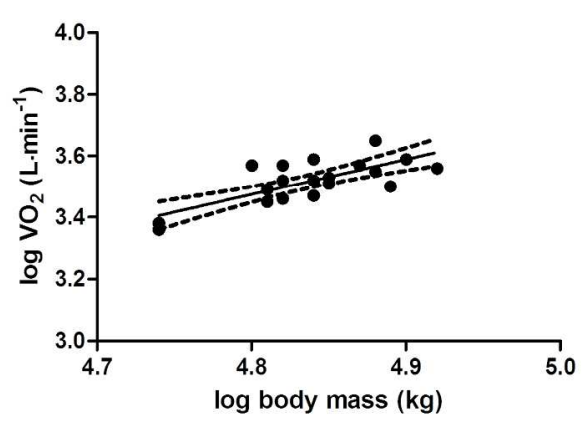

$\mathbf{R}$

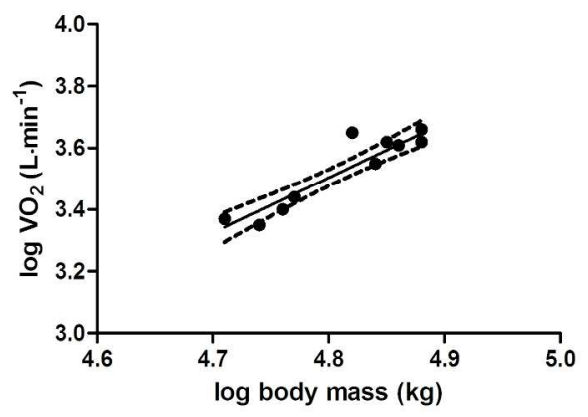

D

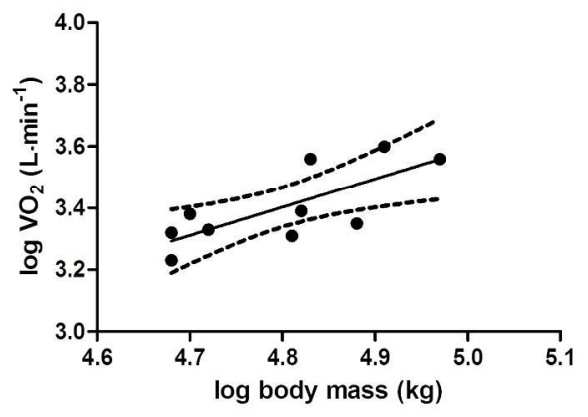

Figure 5. Relationship between oxygen uptake expressed in absolute terms (VO2) and body mass by using allometric log-linear for 3 groups ( $R \& D$, top panel; $R$, middle panel; $D$, bottom panel). Linear regression is shown with a $95 \%$ confidence interval.

$117 \times 268 \mathrm{~mm}(300 \times 300 \mathrm{DPI})$ 